Western University

Scholarship@Western

Physical Therapy Publications

Physical Therapy School

7-10-2019

\title{
Longitudinal Changes in Physical Caregiving for Parents of Children with Cerebral Palsy.
}

Mohammed S Alghamdi

Lisa A Chiarello

Robert J Palisano

Sarah W McCoy

Margo Orlin

See next page for additional authors

Follow this and additional works at: https://ir.lib.uwo.ca/ptpub

Part of the Physical Therapy Commons

Citation of this paper:

Alghamdi, Mohammed S; Chiarello, Lisa A; Palisano, Robert J; McCoy, Sarah W; Orlin, Margo; and Abd-Elkafy, Ehab M,

"Longitudinal Changes in Physical Caregiving for Parents of Children with Cerebral Palsy." (2019). Physical Therapy Publications. 48.

https://ir.lib.uwo.ca/ptpub/48 
Authors

Mohammed S Alghamdi, Lisa A Chiarello, Robert J Palisano, Sarah W McCoy, Margo Orlin, and Ehab M Abd-Elkafy 


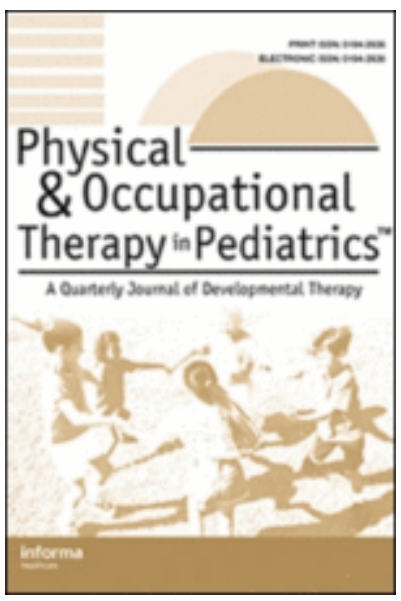

\section{Longitudinal Changes in Physical Caregiving for Parents of Children with Cerebral Palsy}

\begin{tabular}{|r|l|}
\hline Journal: & Physical \& Occupational Therapy In Pediatrics \\
\hline Manuscript ID & WPOP-2019-0007.R1 \\
\hline Manuscript Type: & Original Research \\
\hline Keywords: & $\begin{array}{l}\text { Physical caregiving, cerebral palsy < Populations, motor function, } \\
\text { longitudinal }\end{array}$ \\
\hline \multicolumn{2}{|l}{} \\
\hline
\end{tabular}

\section{SCHOLARONE}

Manuscripts 


\section{Longitudinal Changes in Physical Caregiving for Parents of Children with Cerebral Palsy}

\section{ABSTRACT}

Aims: To determine changes in physical caregiving for parents of children with cerebral palsy (CP) over a two-year period based on children's gross motor function level and age.

Methods: 153 parents of children with CP rated their physical caregiving using the Ease of Caregiving for Children three times over two years. Parents and assessors classified children's gross motor function using the Gross Motor Function Classification System (GMFCS). Physical caregiving was compared at three test times among parents of children grouped by GMFCS level (I, II-III, and IV-V) and age (1.7-5.9 and 6-11 years) using a three-way mixed ANOVA. Results: Among all analyses, a two-way interaction was found between children's GMFCS level and test time on ease of caregiving, $\mathrm{p}<0.01$. Change over two-year period was found for parents of children in level I and II-III, $\mathrm{p}<0.01$, but not parents of children in levels IV-V. At each test time, parents of children in level I reported the greatest ease of caregiving followed by parents of children in levels II-III, and levels IV-V, who reported the lowest ease of caregiving, $p<0.001$. Conclusions: Findings support evaluation and monitoring of physical caregiving for parents of children with CP over time.

KEYWORDS: Physical caregiving; cerebral palsy; motor function; longitudinal 
Family-Centered Care (FCC) is a widely endorsed service delivery model in pediatric rehabilitation that recognizes children and their families during provision of services (King \& Chiarello et al., 2014). Despite the emphasis on FCC, parents' needs may be overlooked as services are primarily focused on addressing children's needs (King et al., 2017). Evidence suggests that parental caregiving of children with disabilities, including cerebral palsy $(\mathrm{CP})$, is associated with poor physical health (Murphy et al., 2007), psychological problems (Sawyer et al., 2011), social problems (Huang et al., 2012), low income, and reduced availability to work in full time jobs (Raina et al., 2004; Wijesinghe et al., 2014). These challenges are commonly referred to in the literature as caregiving burden. The vast majority of research has been focused on understanding parental caregiving burden and less attention has been focused on understanding parental physical caregiving. Alghamdi and colleagues (in press) defined parental physical caregiving as "the physical assistance that parents provide to their children to fulfill daily life activities" (p. x).

Physical caregiving is an important outcome for families of children with CP (Ward et al., 2014). Children with CP often need physical assistance from their parents for mobility and for self-care activities such as feeding, dressing, and toileting (Østensjø et al., 2003). Two aspects of the child, gross motor function and age, consistently have been identified to have an impact on physical caregiving. Greater limitations in gross motor function were found to be associated with high physical and psychological burden, increased time and physical demands, and reduced ease of caregiving (Alghamdi et al., in press; Andrews et al., 2013; Hwang et al., 2011; Palisano et al., 2014; Rassafiani et al., 2012; Sawyer et al., 2011; Svedberg et al., 2010; Ward et al., 2014).

Findings on the impact of children's age on physical caregiving are mixed. Four studies found that older age was associated with more challenging physical caregiving (Andrews et al., 2013; Hwang et al., 2011; McCann, Bull, \& Winzenberg, 2012). Two studies found that younger age 
was associated with more challenging physical caregiving (Crowe \& Florez, 2006; Tadema \& Vlaskamp, 2010). Most recently, Alghamdi et al. (in press) reported that ease of caregiving for parents of children with CP aged 1.5 to 11 years did not vary based on children's age. Methodological variability across studies (e.g. children's age groups and measurement of physical caregiving) might have contributed to mixed findings on the impact of children's age on physical caregiving.

Parent factors impacting physical caregiving of children have not been studied extensively. Parent self-efficacy, socioeconomic status, and perception of support were negatively associated with caregiving demands (Marrón et al., 2013; Wijesinghe et al., 2014). In contrast, parent's mental health problems (e.g. depression) were positively associated with caregiving demands (Sawyer et al., 2011). Knowledge of parent factors impacting physical caregiving may have implications for the provision of family-centered services to children with CP and their families.

Parental caregiving for children with $\mathrm{CP}$ is an ongoing process that changes over time in response to child development and family functioning (Majnemer et al., 2012). To our knowledge, evidence about change over time in physical caregiving for parents of children with CP is limited to one study. For parents of children with CP aged 1.5 to 5 years, Palisano and colleagues (2014) found small positive changes in ease of caregiving over a one-year period among parents of children with higher gross motor function but not among parents of children with lower gross motor function. This study highlighted the importance of monitoring ease of caregiving over time for addressing parents' needs and for future planning. However, the follow-up was limited to one year and only included parents of children with CP under 5 years of age.

To optimize parental physical caregiving, we need to understand how it changes over an extended period of time to better inform therapists about whom services may be indicated and to 
provide parents with anticipatory guidance for future planning. The purpose of this study, therefore, was to determine changes in physical caregiving for parents of children with CP (1.7 to 11 years of age) over a two-year period based on children's gross motor function level and age. The term "ease of caregiving" was used to describe physical caregiving whereas greater ease represents less difficulty in caregiving and lower ease represent more difficulty. We hypothesized that the ease of caregiving will improve over the two-year period and the extent of the improvement will be greatest for parents of older children ( 6 to 11 years of age) who walk without limitations (level I on the Gross Motor Function Classification System (GMFCS), Palisano et al., 2008), followed by parents of younger children (1.7 to 5.9 years of age) who walk without limitations (level I), parents of older children who walk with limitations or support (levels II and III), and parents of younger children who walk with limitations or support (levels II and III). We also hypothesized that the ease of caregiving for parents of younger and older children whose selfmobility is limited (levels IV and V) will not change over the two-year period. Knowledge of change over time would provide therapists with evidence-based information about the pattern of change in physical caregiving, grouped by children's gross motor function level and age, to tailor services and supports to meet parents' needs related to physical caregiving.

\section{METHODS}

\section{Design}

This is a secondary analysis of longitudinal data from 


\section{Participants}

The participants were a subset of 153 children with $\mathrm{CP}$ and their parents who participated in the Study. Participants were recruited between April 2013 to September 2016 from three regions in the United States (Greater Philadelphia, PA; Greater Atlanta, GA; Greater Seattle, WA). The inclusion criteria were 1) children with a primary diagnosis of $\mathrm{CP}$ or gross motor delay, 2) caregivers aged 18 years and older who were able to understand English or Spanish, 3) caregivers who completed the Ease of Caregiving for Children measure 3 times, with a minimum of 18 -month gap between the first and last time. The exclusion criteria were 1) children with diagnoses other than $\mathrm{CP}$ or gross motor delay 2) children who were wards of the state, and 3) caregivers who did not speak English or Spanish. Ethical approval was obtained from each university's Institutional Review Boards and from Institutional Review Boards of sites where data collection or recruitment took place, if required. Prior to the data collection, written informed consent was obtained from parents and assent was obtained from children when applicable.

Demographic information about children and their caregivers is presented in Table 1. Children were 1.7 to 11 years of age, $56.2 \%$ were boys, and $70.7 \%$ were white. Caregivers had a mean age of $39.7(\mathrm{SD}=7.1)$ years, $88.8 \%$ were mothers, $80.7 \%$ were white, and $83 \%$ had an education level of greater than high school. Given that the majority of caregivers were mothers and fathers (95.4\%), the term "parents" was used throughout this paper.

\section{Measures}

The Ease of Caregiving for Children (Alghamdi et al., in press; Ward et al., 2014) is a 12-item 
parent-report measure of physical caregiving for parents of children with CP between 1.5 to 11 years of age. Parents rate their perceived level of difficulty when providing physical assistance to their children on a 5-point Likert scale (1= "very difficult" to 5= "no help is needed"). Parents are instructed to consider their safety, confidence, and the physical and time demands of the tasks (e.g. child's mobility, positioning, self-care activities) when rating the level of difficulty. Rasch analysis provided evidence on the unidimensionality of the measure, logical ordering of the items based on task difficulty, and appropriateness of the item responses (Alghamdi et al., in press; Ward et al., 2014). The measure demonstrated high internal consistency (Cronbach's alpha was 0.92) (Ward et al., 2014) and excellent test-retest reliability with an interval of 4 weeks between test and retest measurements, $\left(\operatorname{ICC}_{(2,1)}=0.75,95 \%\right.$ CI $\left.[0.57-0.86]\right)$ (Alghamdi et al. in press). The measure's ability to discriminate physical caregiving for parents of children with varying functional abilities supported the construct (known groups) validity (Ward et al., 2014; Alghamdi et al., in press). The measure's ability to detect change over one year was supported for parents of young children with higher gross motor functions (GMFCS level I-III) but not for parents of children in GMFCS IV-V (Palisano et al., 2014). Total scores vary between 0 and 100 with higher scores indicating greater ease of caregiving (i.e. less difficulty in caregiving).

The Gross Motor Function Classification System (GMFCS) is used to classify gross motor function of children with CP into one of five levels based on self-initiated movement with emphasis on sitting, transferring, and mobility (Palisano et al., 2008). Moderate $(\kappa=0.55)$ and substantial $(\kappa=0.75)$ inter-rater reliability were reported for children younger than 2 years and children older than 2 years of age, respectively (Palisano et al., 1997). Content and construct validity of the GMFCS have been supported (Palisano et al., 1997; Palisano et al., 2008). 


\section{Procedures}

Parents completed the Ease of Caregiving for Children measure three times over two years as part of their participation in the Study. The second measurement was completed 7 to 16 months after the first time point $(\mathrm{M}=12.7, \mathrm{SD}=1.4)$. The time gap between the first and third measurements varied from 18 to 28 months $(\mathrm{M}=23.1, \mathrm{SD}=2.6)$. Parents and assessors independently classified children's gross motor function at the first data collection point in the

Study and then classified children approximately 12 months later. The first classification was used for children 2 years of age and older. For children younger than 2 years of age, the second classification was used (McCoy et al., 2018) as inter-rater reliability of the GMFCS is lower for children less than 2 years of age (Palisano et al., 2008). When a parent and assessor disagreed on the classification level, discussion was initiated to reach a consensus. Parents' and assessors' classifications initially matched or consensus was reached $97.4 \%$ of the time. In cases where consensus was not reached, the parent's classification was used unless the assessor provided specific information to support a different classification (Bartlett et al., 2016).

\section{Data Analysis}

Statistical analyses were conducted using SPSS software (SPSS Statistics for Windows, Version 24.0. Armonk, NY: IBM Corp.). Descriptive statistics were computed for all variables. Ease of Caregiving raw scores (ordinal level data) were converted to interval level data using a scoring system reported by Alghamdi et al. (in press). The scoring system was devised from Rasch analysis of the measure in which the sum score of all items was converted to an interval scale of 0 to 100 (Alghamdi et al., in press).

Our initial intent was to conduct a mixed three-way analysis of covariance (ANCOVA) to examine the effect of GMFCS level, children's age, and test time on physical caregiving while 
accounting for selective parent demographics as covariates. Covariates examined included parent's employment status, level of education, income, and household size. Correlations between the four covariates and the dependent variable (Ease of Caregiving score) were low and not significant ( $\mathrm{r}$ $=-0.11$ to $0.14, p>0.05)$. Consequently, a mixed three-way analysis of variance (ANOVA) was used. The mixed three-way ANOVA design consisted of two between-subject factors and one within-subject factor. Between-subject factors were: 1) children's GMFCS level (3 levels: level I; II-III; and IV-V); and 2) children's age (2 levels: younger children [1.7- 5.9 years]; older children [6 -11 years]. The Within-subject factor was test time and consisted of 3 measurements of physical caregiving (Time 1, 2, and 3). Evaluation of outliers, normality, homogeneity of variance, and sphericity met the assumptions for three-way mixed ANOVA.

Partial eta-squared $\left(\eta^{2}\right)$ was calculated to estimate the effect size for the ANOVA analyses and was interpreted as follows: $\eta^{2}=0.01$ indicates a small effect, $\eta^{2}=0.06$ a medium effect, and $\eta^{2}=$ 0.14 a large effect size (Lakens, 2013). Cohen's $d z$, an effect size statistic for correlated measurements, was calculated to quantify the magnitude of change in physical caregiving between three time points over two years (Lakens, 2013). Cohen's $d z$ and associated 95\% confidence interval were calculated using SPSS syntax (Wuensch, 2012). Cohen's $d z$ can be interpreted by the following conventions: $d z=0.2$ indicates a small effect, $d z=0.5$ indicates medium effect, and $d z=0.8$ indicates a large effect (Lakens, 2013). An alpha level of 0.05 was used for all analyses unless otherwise stated.

\section{RESULTS}

Descriptive statistics for parental physical caregiving by children's GMFCS level and age group across time points are presented in Table 2. The three-way interaction among children's GMFCS level, age group, and time on physical caregiving was not significant $\left(F_{(3.78,277.56)}=0.62, p=0.64\right.$, 
partial $\eta^{2}=0.008$ ). Similarly, neither the two-way interaction between children's GMFCS level and age $\left(F_{(2,147)}=0.9, p=0.41\right.$, partial $\left.\eta^{2}=0.01\right)$ nor the two-way interaction between children's age and time $\left(F_{(1.89,277.56)}=1.5, p=0.23\right.$, partial $\left.\eta^{2}=0.01\right)$ on physical caregiving was significant. There was no significant main effect of age on physical caregiving $\left(F_{(1,147)}=3.3, p=0.07\right.$, partial $\left.\eta^{2}=0.02\right)$

There was a significant two-way interaction between children's GMFCS level and time on physical caregiving $\left(F_{(3.78,277.56)}=3.9, p=0.005\right.$, partial $\left.\eta^{2}=0.051\right)$. Main effects were found for both children's GMFCS level $\left(F_{(2,147)}=81.9, p<0.001\right.$, partial $\left.\eta^{2}=0.53\right)$ and time $\left(\mathrm{F}_{(1.89,277.56)}=\right.$ $8.7, p<0.001$, partial $\left.\eta^{2}=0.056\right)$. For the effect of children's GMFCS level, using an adjusted alpha level of $p<0.017$, significant simple main effects were found for children's GMFCS level on physical caregiving at Time $1\left(F_{(2,147)}=53.9, p<0.001\right.$, partial $\left.\eta^{2}=0.42\right)$; Time $2\left(F_{(2,147)}=\right.$ 50.9, $p<0.001$, partial $\left.\eta^{2}=0.41\right)$; and Time $3\left(F_{(2,147)}=84.2, p<0.001\right.$, partial $\left.\eta^{2}=0.53\right)$. Across all time points, pairwise comparisons indicated that parents of children in level I reported the greatest ease of caregiving, followed by parents of children in levels II-III, and by parents of children in level IV-V who reported the lowest ease of caregiving, $p<0.001$.

For the effect of test time on physical caregiving, using an adjusted alpha level of $p<0.017$, significant differences were found for parents of children in level I and levels II-III. Parents of children in level I and levels II-III reported greater ease of caregiving at Time 2 compared to Time $1, p<0.01$, and the effect size was small ( $d z=0.43$ and 0.35 , respectively). Additionally, parents of children in level I reported greater ease of caregiving at Time 3 compared to Time $1, p<0.001$, and the effect size was medium $(d z=0.54)$. Parents of children in level IV-V reported no change in ease of caregiving over the two-year period, $p>0.05$; however, the effect size calculation showed a decline (small effect, $d z=-0.27$ ) in ease of caregiving from Time 2 to Time 3. The 
magnitude of change in ease of caregiving over time for children in each GMFCS level is depicted in Figure 1 and presented in Table 3.

\section{DISCUSSION}

The findings suggest that children's gross motor function level, but not age, influenced change in ease of caregiving for parents of children with CP (1.7 - 11 years of age $)$ over the two-year period, partially supporting our hypotheses. As expected, the ease of caregiving improved over time (i.e. less difficulty in caregiving) for parents of children classified in levels I-III whereas the ease of caregiving for parents of children in levels IV-V did not. Our findings for change over time are comparable to and expand the findings reported by Palisano et al. (2014) for parents of children with $\mathrm{CP}$ under 5 years of age. The magnitude of improvement in ease of caregiving for parents of children classified in level I was of small effect at one year and increased to a medium effect at two years. It is plausible that children's increased functional independence contributed to the observed improvement in ease of caregiving. The magnitude of improvement in ease of caregiving for parents of children classified in levels II-III was of small effect at one year and plateaued afterwards. The plateau effect may reflect parental adaptation to providing physical assistance to children who may have reached their potential in mobility and self-care. For parents of children classified in levels IV-V, the lack of change in ease of caregiving over the two-year period may reflect children's persistent need for physical assistance from their parents due to their functional limitations. Although there was a small decline, as noted by effect size, in ease of caregiving over the second year, we do not believe this decline is clinically meaningful.

Change over time in ease of caregiving might be secondary to changes in child, parent, caregiving task, and environmental attributes. Children with $\mathrm{CP}$ exhibit changing needs in daily activities across their lifespan and consequently may require various levels of assistance or 
supervision to participate in daily activities. The changing nature of children's needs may, therefore, contribute positively or negatively to the observed change in physical caregiving. Although not examined in our study, parents' attributes contributing to the change in ease of caregiving may include physical and psychological health, adaptation, and physical capacity to meet their children's evolving needs. Finally, task and environmental attributes, such as complexity of caregiving task and context, and availability of supports and services, may also explain change over time in ease of caregiving.

The lack of differences in ease of caregiving between children 1.7 to 5.9 years of age and children 6 to 11 years of age do not necessarily indicate that children's age has no effect on ease of caregiving. In our study, age-related differences in the ease of caregiving might not have been captured due to the relatively younger age of children. Children's aging is typically associated with increased physical size and acquisition/deterioration of functional abilities, which consequently could have positive or negative impact on ease of caregiving. In addition, children's preferences and interests in activities and participation change as they become older and this could influence the ease of caregiving. In a related study, we postulated that age-related differences in ease of physical caregiving may appear later in childhood during onset of puberty and related physical growth (Alghamdi et al., in press).

As expected, ease of caregiving varied based on children's gross motor function level indicating the impact of children's functional limitations on physical caregiving. Our finding of the influence of children's gross motor function on ease of caregiving is consistent with previous research (Ward et al., 2014; Hwang et al., 2011; Østensjø et al., 2003). A notable finding in our study is that parents of children classified in level I, on average, provided no physical assistance to their children to move and perform self-care activities. In contrast, parents of children classified 
in levels II-III and IV-V, on average, provided physical assistance to their children to move and perform self-care activities with no difficulty and little difficulty, respectively. These findings might be related to children's performance in self-care activities. Fiss et al. (2016) reported that self-care performance of children with CP (1.5 to 12 years of age) varied across GMFCS levels. Children with higher gross motor function participated more independently in self-care activities than children with lower gross motor function (Fiss et al., 2016). Additionally, the reported level of difficulty by parents in our study (little difficulty to no assistance needed) may reflect their successful adaptation strategies and their positive approach of caring for their children.

A great variability was found in ease of caregiving for parents of children with CP with parents rating their physical caregiving from very difficult to no assistance provided to children. Variability in ease of caregiving was anticipated given the heterogeneity of children with $\mathrm{CP}$ and the multifactorial nature of physical caregiving. In addition, the variability may be attributed to the subjective nature of the Ease of Caregiving for Children measure. The measure asks parents to rate their "perceived" level of difficulty when providing physical assistance to their children in mobility and self-care activities. Factors such as cultural values and beliefs are likely to influence parents' perception of difficulty and consequently their ratings of the difficulty of caregiving.

\section{Implications for practice}

We believe that physical and occupational therapists have a role in optimizing the caregiving experience of parents of children with CP. The variability of ease of caregiving reported by parents based on their children's gross motor function level and age highlights the importance of individualization of the evaluation process, goal setting, and plan of care for parents and their children. We recommend that therapists routinely ask parents' about their concerns and needs related to physical caregiving and encourage the use of validated parent self-reported measures 
such as the Ease of Caregiving for Children. If parents are experiencing difficulty in physical caregiving, we recommend discussion of whether difficulty is due to physical demands, concerns for safety, availability of supports and resources, confidence in their abilities, or some combination.

The model of family-oriented services by King et al. (2017) has implications for therapists' clinical decision making to meet the needs of parents and their children. The model offers a range of strategies to support parental caregiving including, but not limited to, information sharing, parent education, and service coordination. Parents of children with $\mathrm{CP}$ expressed a need for information for future planning (Almasri et al., 2018; Palisano et al., 2010). We, therefore, encourage therapists to provide anticipatory guidance to parents of children with $\mathrm{CP}$, especially children in GMFCS levels IV-V. For example, parents of children in GMFCS levels IV-V might consider adaptive equipment for positioning and mobility and respite care for their own wellbeing.

\section{Limitations and future directions}

Although parents were followed for a two-year period, it is plausible that the timeframe was not long enough to detect large differences in physical caregiving for parents of children with $\mathrm{CP}$. We did not account for other factors related to the child (e.g. adaptive behavior), parent (e.g. selfefficacy and mental health), environment (e.g. parental supports), and services (e.g. child- and parent-focused interventions) that could potentially influence parental physical caregiving. Our study included a relatively small number of participants across combinations of GMFCS and age groups. We did not include parents of adolescents with CP for whom ease of physical caregiving might be affected by physical size. Future research is warranted to examine a comprehensive model of child, parent/family, and environment, and service determinants of physical caregiving 
for parents of children and youth with CP. In addition, research is needed to develop and examine the effect of interventions and services specifically directed to parental physical caregiving.

\section{CONCLUSION}

Children's gross motor function level, but not age, was found to influence change in ease of caregiving for parents of children with CP (1.7 - 11 years of age) over a two-year period. Parents of children classified in GMFCS level I and levels II-III reported greater ease of caregiving over two years with a medium and small effects, respectively. However, no change in the ease of caregiving over two years was found for parents of children classified in levels IV-V. At each time point, parents of children with higher gross motor function reported greater ease of caregiving compared to parents of children with lower gross motor function; however, there was variability within motor function levels. In addition, no difference in ease of caregiving was found between parents of children $<6$ years and $\geq 6$ years. The variability in ease of caregiving and change over time for parents of children with $\mathrm{CP}$ highlights the importance of individualization of the evaluation process, goal setting, and the plan of care. 


\section{REFERENCES:}

Alghamdi, MS., Chiarello, LA., Avery, L., Palisano, RJ. (in press). Ease of Caregiving for Children: re-validation of psychometric properties of the measure for children with cerebral palsy up to 11 years of age. Developmental Neurorehabiliation.

Almasri, N. A., An, M., \& Palisano, R. J. (2018). Parents' perception of receiving familycentered care for their children with physical disabilities: a meta-analysis. Phys Occup Ther Pediatr, 38(4), 427-443.

Andrews, M., Bolt, D. M., Braun, M., \& Benedict, R. E. (2013). Measuring exertion during caregiving of children and young adults with cerebral palsy who require assistance for mobility and self-care. Phys Occup Ther Pediatr, 33(3), 300-312. doi:10.3109/01942638.2012.754395

Crowe, T.K., \& Florez, S.I. (2006). Time use of mothers with school-age children: A continuing impact of a child's disability. American Journal of Occupational Therapy, 60(2), 194-203.

Fiss, A., Chiarello, L., \& McCoy, S. (2016). Reliability and validity of a revised version of the self-care domain of the Child Engagement in Daily Life Measure. Paper presented at the IV STEP Conference, American Physical Therapy Association, Columbus, OH. URL: https://u.osu.edu/ivstep/poster/abstracts/024_laforme-fiss-et-al/

Huang, YP., Kellett, U., \& St John, W. (2012). Being concerned: caregiving for Taiwanese mothers of a child with cerebral palsy. Journal of Clinical Nursing, 21(1-2), 189-197.

Hwang, M., Kuroda, M. M., Tann, B., \& Gaebler-Spira, D. J. (2011). Measuring care and comfort in children with cerebral palsy: the care and comfort caregiver questionnaire. American Academy of Physical Medicine \& Rehabilitation, 3(10), 912-919. doi:10.1016/j.pmrj.2011.05.017 
Hanna, S. E., Rosenbaum, P. L., Bartlett, D. J., Palisano, R. J., Walter, S. D., Avery, L., \& Russell, D. J. (2009). Stability and decline in gross motor function among children and youth with cerebral palsy aged 2 to 21 years. Developmental Medicine \& Child Neurology, 51(4), 295-302.

King, G., Williams, L., and Hahn Goldberg, S. (2017) Family-oriented services in pediatric rehabilitation: a scoping review and framework to promote parent and family wellness. Child: Care, Health and Development, 43: 334-347. doi: 10.1111/cch.12435.

King, G., \& Chiarello, L. (2014). Family-centered care for children with cerebral palsy: conceptual and practical considerations to advance care and practice. Journal of Child Neurology, 29(8), 1046-1054. doi:10.1177/0883073814533009

Lakens, D. (2013). Calculating and Reporting Effect Sizes to Facilitate Cumulative Science: A Practical Primer for t-tests and ANOVAs. Frontiers in Psychology, 4. doi:10.3389/fpsyg.2013.00863

McCoy, SW., Bartlett, D., Smersh, M., Galuppi, B., \& Hanna, S., Collaboration Group: On Track Study Team (March 2018). Monitoring development of children with cerebral palsy: the On Track study. Protocol of a longitudinal study of development and services. Available at: https://www.canchild.ca/en/resources/294-monitoring-development-of-children-withcerebral-palsy-the-on-track-study-protocol-of-a-longitudinal-study-of-development-and$\underline{\text { services }}$

Marrón, Elena M, Redolar-Ripol, Diego, Boixadós, Mercè, Nieto, Rubén, Guillamón, Noemí, Hernández, Eulália, \& Gómez, Benigna. (2013). Burden on caregivers of children with cerebral palsy: predictors and related factors. Universitas Psychologica, 12(3), 767-777. 
McCann, Damhnat, Bull, Rosalind, \& Winzenberg, Tania. (2012). The daily patterns of time use for parents of children with complex needs: A systematic review. Journal of Child Health Care, 1367493511420186.

Majnemer, A., Shevell, M., Law, M., Poulin, C., \& Rosenbaum, P. (2012). Indicators of distress in families of children with cerebral palsy. Disability and Rehabilitation, 34(14), 1202-1207.

Murphy, Nancy A, Christian, Becky, Caplin, Deidre A, \& Young, Paul C. (2007). The health of caregivers for children with disabilities: caregiver perspectives. Child: Care, Health and Development, 33(2), 180-187.

Østensjø, S., Carlberg, E. B., \& Vøllestad, N. K. (2003). Everyday functioning in young children with cerebral palsy: functional skills, caregiver assistance, and modifications of the environment. Developmental medicine and child neurology, 45(9), 603-612.

Palisano, R. J., Rosenbaum, P., Bartlett, D., \& Livingston, M. H. (2008). Content validity of the expanded and revised Gross Motor Function Classification System. Developmental Medicine and Child Neurology, 50(10), 744-750. doi:10.1111/j.1469-8749.2008.03089.x

Palisano, R. J., Almarsi, N., Chiarello, L. A., Orlin, M. N., Bagley, A., \& Maggs, J. (2010). Family needs of parents of children and youth with cerebral palsy. Child: care, health and development, 36(1), 85-92.

Palisano, R.J., Chiarello, L.A., McCoy, S.W., Bartlett, D., \& An, M. (2014). Use of the Child Engagement in Daily Life and Ease of Caregiving for Children to Evaluate Change in Young Children with Cerebral Palsy. Phys Occup Ther Pediatr. doi:10.3109/01942638.2014.907221 Raina, P., O'Donnell, M., Schwellnus, H., Rosenbaum, P., King, G., Brehaut, J., . . Wood, E. (2004). Caregiving process and caregiver burden: conceptual models to guide research and practice. BMC Pediatr, 4, 1. doi:10.1186/1471-2431-4-1 
Rassafiani, M, Kahjoogh, M.A., Hosseini, A, \& Sahaf, R. (2012). Time use in mothers of children with cerebral palsy: a comparison study. Hong Kong Journal of Occupational Therapy, 22(2), 70-74.

Sawyer, M.G., Bittman, M., La Greca, A.M., Crettenden, A.D., Borojevic, N, Raghavendra, P, \& Russo, R. (2011). Time demands of caring for children with cerebral palsy: what are the implications for maternal mental health? Developmental Medicine \& Child Neurology, 53(4), 338-343.

Svedberg, L.E., Englund, E, Malker, H, \& Stener-Victorin, E. (2010). Comparison of impact on mood, health, and daily living experiences of primary caregivers of walking and non-walking children with cerebral palsy and provided community services support. European Journal of Paediatric Neurology, 14(3), 239-246.

Tadema, A.C., \& Vlaskamp, C. (2010). The time and effort in taking care for children with profound intellectual and multiple disabilities: a study on care load and support. British Journal of Learning Disabilities, 38(1), 41-48.

Ward, K., Chiarello, L.A., Bartlett, D., Palisano, R.J., McCoy, S.W., \& Avery, L. (2014). Ease of Caregiving for Children: a measure of parent perceptions of the physical demands of caregiving for young children with cerebral palsy. Research in developmental disabilities, $35(12), 3403-3415$.

Wijesinghe, C. J, Cunningham, N, Fonseka, P, Hewage, C.G., \& Østbye, T. (2014). Factors associated with caregiver burden among caregivers of children with cerebral palsy in Sri Lanka. Asia-Pacific Journal of Public Health, 1010539514548756.

Wuensch, K. L. (2012). Using SPSS to obtain a confidence interval for Cohen's d. Retrived from: http://core.ecu.edu/psyc/wuenschk/SPSS/CI-d-SPSS.pdf. 


\begin{tabular}{|c|c|c|}
\hline Variable & Description & $\mathrm{N}(\%)^{* *}$ \\
\hline $\begin{array}{l}\text { Child age in years } \\
(\mathrm{n}=153)\end{array}$ & Mean (SD) & $6.5(2.7)$ \\
\hline \multirow[t]{2}{*}{$\operatorname{Sex}(n=153)$} & Boy & $86(56.2)$ \\
\hline & Girl & $67(43.8)$ \\
\hline \multirow{2}{*}{$\begin{array}{l}\text { Child Ethnicity } \\
(\mathrm{n}=152)^{*}\end{array}$} & Non-Hispanic & $137(90.1)$ \\
\hline & Hispanic & $15(9.9)$ \\
\hline \multirow{4}{*}{ Child Race $(\mathrm{n}=150)^{*}$} & Asian & $7(4.7)$ \\
\hline & Black/African American & $24(16)$ \\
\hline & White & $106(70.7)$ \\
\hline & Multiracial & $13(8.7)$ \\
\hline \multirow[t]{5}{*}{ Child GMFCS $(\mathrm{n}=153)$} & Level I & $44(28.8)$ \\
\hline & Level II & $35(22.8)$ \\
\hline & Level III & $16(10.5)$ \\
\hline & Level IV & $28(18.3)$ \\
\hline & Level V & $30(19.6)$ \\
\hline \multirow{5}{*}{$\begin{array}{l}\text { Distribution of } \\
\text { Involvement }(n=153)\end{array}$} & Monoplegia & $1(0.7)$ \\
\hline & Hemiplegia & $39(25.5)$ \\
\hline & Diplegia & $32(20.9)$ \\
\hline & Triplegia & $7(4.6)$ \\
\hline & Quadriplegia & $74(48.4)$ \\
\hline Caregiver Age $(\mathrm{n}=145)^{*}$ & Mean (SD) & $39.7(7.13)$ \\
\hline \multirow{2}{*}{$\begin{array}{l}\text { Caregiver Ethnicity } \\
(\mathrm{n}=151)^{*}\end{array}$} & Non-Hispanic & $143(94.7)$ \\
\hline & Hispanic & $8(5.3)$ \\
\hline \multirow{3}{*}{$\begin{array}{l}\text { Caregiver Race } \\
(\mathrm{n}=150)^{*}\end{array}$} & Asian & $6(4.0)$ \\
\hline & Black/African American & $23(15.3)$ \\
\hline & White & $121(80.7)$ \\
\hline \multirow{3}{*}{$\begin{array}{l}\text { Caregiver Relationship to } \\
\text { Child }(\mathrm{n}=152)^{*}\end{array}$} & Mother & $135(88.8)$ \\
\hline & Father & $10(6.6)$ \\
\hline & Others & $7(4.7)$ \\
\hline \multirow{6}{*}{$\begin{array}{l}\text { Total Household Income } \\
(\mathrm{n}=152)^{*}\end{array}$} & $<\$ 30,000$ & $26(17.1)$ \\
\hline & $\$ 30,000-\$ 44,999$ & $10(6.6)$ \\
\hline & $\$ 45,000-\$ 59,999$ & $11(7.2)$ \\
\hline & $\$ 60,000-\$ 74,999$ & $15(9.9)$ \\
\hline & $\geq \$ 75,000$ & $73(48.0)$ \\
\hline & Prefer not to answer & $17(11.2)$ \\
\hline \multirow{3}{*}{$\begin{array}{l}\text { Caregiver Education } \\
(\mathrm{n}=152)^{*}\end{array}$} & High school or less & $26(17.1)$ \\
\hline & Community college degree & $32(21.1)$ \\
\hline & University/graduate degree & $94(61.9)$ \\
\hline \multirow{3}{*}{$\begin{array}{l}\text { Caregiver Employment } \\
(\mathrm{n}=150)^{*}\end{array}$} & Full-time (30 hours or more/week) & $49(32.7)$ \\
\hline & Part-time (less than 30 hours/week) & $32(21.3)$ \\
\hline & Not employed & $69(46.0)$ \\
\hline Household Size $(\mathrm{n}=152)^{*}$ & Median (Min- Max) & $4(2-10)$ \\
\hline
\end{tabular}




\begin{tabular}{|c|c|c|c|c|c|}
\hline & \multicolumn{5}{|c|}{$\begin{array}{l}\text { Table 2. Descriptive Statistics of Ease of Caregiving Scores by Children's GMFCS Level, Age Group and } \\
\text { Time* }\end{array}$} \\
\hline & Age Group & Level I & Levels II-III & Levels IV-V & Total \\
\hline \multirow{3}{*}{ Time 1} & Younger (1.7 to 5.9 years) & $\begin{array}{l}64.5(13.6) \\
n=19\end{array}$ & $\begin{array}{l}52.0(10.0) \\
\mathrm{n}=25\end{array}$ & $\begin{array}{c}41.7(7.6) \\
n=19\end{array}$ & $\begin{array}{c}52.7(13.8) \\
n=63\end{array}$ \\
\hline & Older (6- 11 years) & $\begin{array}{c}73.0(18.4) \\
n=25\end{array}$ & $\begin{array}{l}56.0(14.8) \\
n=26\end{array}$ & $\begin{array}{c}42.4(8.0) \\
n=39\end{array}$ & $\begin{array}{c}54.8(18.4) \\
n=90\end{array}$ \\
\hline & Total & $\begin{array}{l}69.3(16.7) \\
\mathrm{n}=44\end{array}$ & $\begin{array}{c}54.1(12.7) \\
\mathrm{n}=51\end{array}$ & $\begin{array}{c}42.2(7.8) \\
\mathrm{n}=58\end{array}$ & $\begin{array}{c}54.0(16.7) \\
N=153\end{array}$ \\
\hline \multirow{3}{*}{ Time 2} & Younger (1.7 to 5.9 years) & $\begin{array}{c}72.2(17.8) \\
\mathrm{n}=19\end{array}$ & $\begin{array}{c}55.6(16.0) \\
\mathrm{n}=25\end{array}$ & $\begin{array}{c}41.8(12.6) \\
n=19\end{array}$ & $\begin{array}{c}56.4(19.5) \\
n=63\end{array}$ \\
\hline & Older (6- 11 years) & $\begin{array}{c}77.9(18.4) \\
\mathrm{n}=25\end{array}$ & $\begin{array}{c}64.3(21.4) \\
n=26\end{array}$ & $\begin{array}{c}42.2(8.7) \\
n=39\end{array}$ & $\begin{array}{c}58.5(22.0) \\
\mathrm{n}=90\end{array}$ \\
\hline & Total & $\begin{array}{c}75.4(18.2) \\
n=44\end{array}$ & $\begin{array}{c}60.0(19.3) \\
\mathrm{n}=51\end{array}$ & $\begin{array}{c}42.1(10.0) \\
\mathrm{n}=58\end{array}$ & $\begin{array}{c}57.7(21.0) \\
\mathrm{N}=153\end{array}$ \\
\hline \multirow{3}{*}{ Time 3} & Younger ( 1.7 to 5.9 years) & $\begin{array}{c}74.4(15.2) \\
n=19\end{array}$ & $\begin{array}{c}56.6(16.1) \\
n=25\end{array}$ & $\begin{array}{c}40.9(8.0) \\
n=19\end{array}$ & $\begin{array}{c}57.2(18.9) \\
n=63\end{array}$ \\
\hline & Older (6- 11 years) & $\begin{array}{c}77.8(15.0) \\
\mathrm{n}=25\end{array}$ & $\begin{array}{c}59.5(16.7) \\
\mathrm{n}=26\end{array}$ & $\begin{array}{c}39.5(7.9) \\
\mathrm{n}=39\end{array}$ & $\begin{array}{c}55.9(20.5) \\
n=90\end{array}$ \\
\hline & Total & $\begin{array}{c}76.3(15.0) \\
n=44\end{array}$ & $\begin{array}{c}58.1(16.3) \\
n=51\end{array}$ & $\begin{array}{c}40.0(7.9) \\
n=58\end{array}$ & $\begin{array}{c}56.5(19.8) \\
\mathrm{N}=153\end{array}$ \\
\hline
\end{tabular}

* Data are Rasch scores and presented as mean (standard deviation). Higher score indicates greater ease of caregiving. 


\begin{tabular}{|l|l|l|l|}
\hline \multicolumn{4}{|c|}{ Table 3. Magnitude of change in Ease of Caregiving score across time points * } \\
\hline GMFCS level & Time 1 to Time 2 & Time 2 to Time 3 & Time 1 to Time 3 \\
\hline I & $0.43,[0.12-0.73]^{* *}$ & $-0.01,[-0.20-0.39]$ & $0.54,[0.22-0.85]^{* *}$ \\
\hline II-III & $0.35,[0.07-0.63]^{* *}$ & $0.14,[-0.13-0.42]$ & $0.27,[-0.01-0.55]$ \\
\hline IV-V & $0.01,[-0.25-0.27]$ & $-0.27,[-0.006--0.53]^{* *}$ & $-0.26,[-0.005-0.52]$ \\
\hline
\end{tabular}

* Data are Cohen's $d z$ and 95\% Confidence Interval

$* *$ Cohen's $d z$ is significant at $\mathrm{p}<0.05$ 


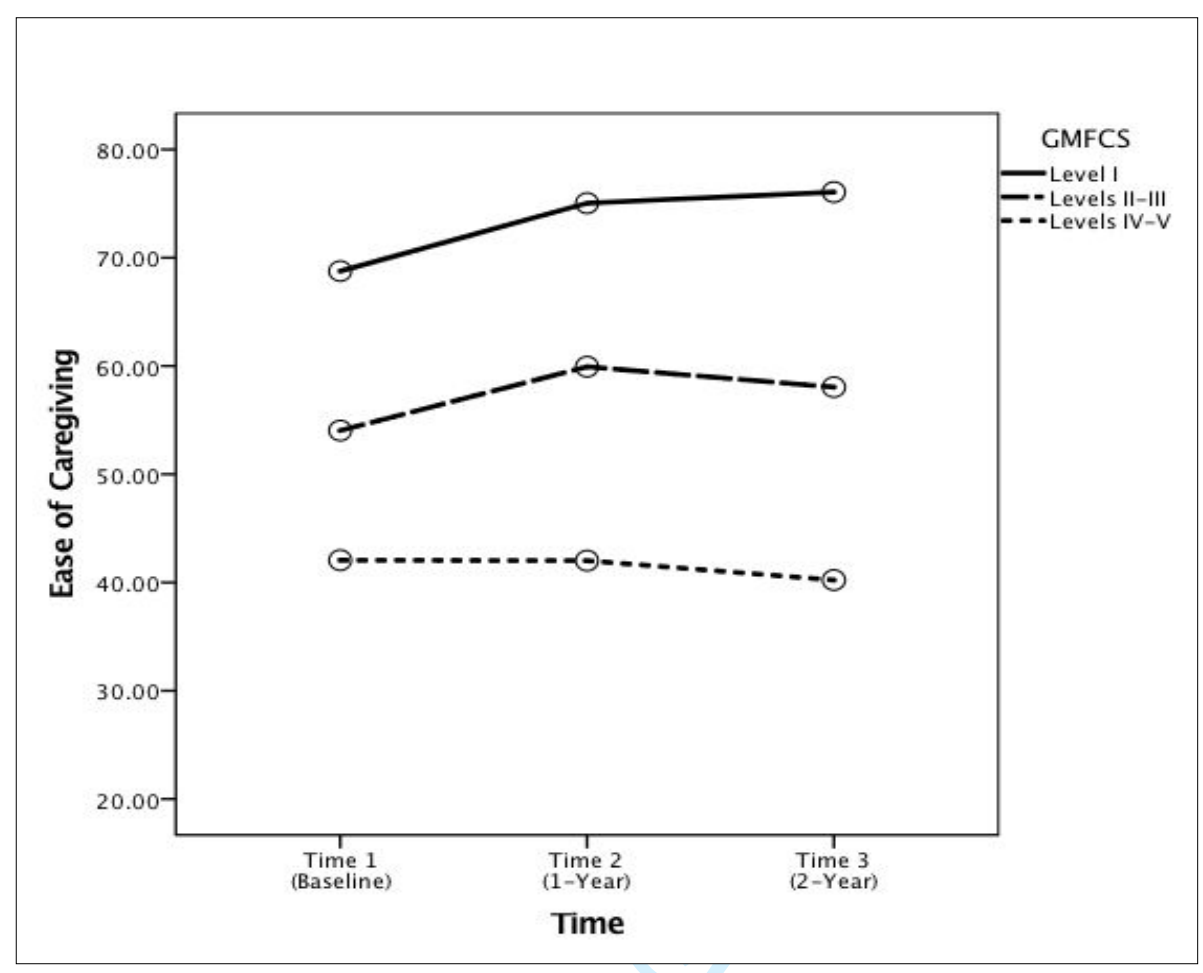

Figure 1. Ease of Caregiving scores over time based on GMFCS level 